\title{
Téoros
}

Revue de recherche en tourisme

\section{Comment Montréal est devenu Paris pour les Américains!}

\section{Marie-Janou Lusignan}

Volume 14, numéro 2, été 1995

Le tourisme : toute une histoire!

URI : https://id.erudit.org/iderudit/1075096ar

DOI : https://doi.org/10.7202/1075096ar

Aller au sommaire du numéro

Éditeur(s)

Université du Québec à Montréal

ISSN

0712-8657 (imprimé)

1923-2705 (numérique)

Découvrir la revue

Citer cet article

Lusignan, M.-J. (1995). Comment Montréal est devenu Paris pour les Américains! Téoros, 14(2), 20-23. https://doi.org/10.7202/1075096ar d'utilisation que vous pouvez consulter en ligne.

https://apropos.erudit.org/fr/usagers/politique-dutilisation/ 


\title{
Comment Montréal est devenu Paris pour les Américains!
}

\author{
Marie-Janou Lusignan*
}

\section{* De toutes les villos en Amérique, Montréal est celle qui m'a séduit le phus..." Jules Leclercq, voyageur français, 1886.}

En 1994, l'Office des congrès et du tourisme du Grand Montréal (OCTGM) célébrait ses 75 ans de fondation. Dans le cadre des activités marquant cet anniversaire, le Centre d'histoire de Montréal présentait, du 21 septembre 1994 au 16 avril 1995, une exposition intitulée Suivez Le guide' L'bitoire du tourime à Montréal. Peu d'historiens se sont intéressés au sujet et la réalisation de cette exposition a nécessitế de nombreuses recherches documentaires et iconographiques afin de faire sortir des méandres de l'oubli, les grands jalons de l'histoire du tourisme à Montréal. Les faits marquants de cette histoire sont ici présentés.

\section{Le premier touriste à Ville-Marie}

Ville-Marie venait-elle à peine d'être fondée par le sieur de Maisonneuve, qu'un jeune normand, du nom de Asseline de Ronval, sans doute le premier touriste en terre néo-française, foula son sol. Contrairement à la plupart des voyageurs qui venaient en Nouvelle-France afin de remplir des missions économiques ou politiques, Asseline de Ronval ne voyageait que pour son agrément. Animé par la curiosité et la soif de découverte, c'est en mai 1662 que le jeune homme s'embarqua à bord d'un vollier en direction de Québec. Le voyage dura plus d'un mois. A peine venait-1] d'atteindre le Nouveau-Monde qu'il fut le témoin d'une scène horrifiante qui le hanta tout au long de son voyage: à Tadoussac, un jeune homme fut scalpé devant ses yeux.

Après avoir séjourné à Québec et à TroisRivières, Asseline de Ronval visita Montréal. C'étrait alors, sclon lui, une bien petite

\footnotetext{
- Madame Marie-Janou Lusignañ est professeute audepartement de tourisme du Cegep de Granby. Haute-Yamaska et a été chargée de projet pour lexposition: Survez le guide / L his loure dutounsme a Montreal
}

ville qui avait peu d'aturactions et de distractions à offrir à ses visiteurs: quelques rues et l'Hôtel-Dieu qui induait la maison de Jeanne Mance, la chapelle et l'hôpital ${ }^{(1)}$. Son séjour dedeux semaines dans la ville lui permit néanmoins de rencontrer tous les notables.

\section{[...] nous poursuivisme nostre voyage du montreal non pas sans grande peinne car le courant de leau nous estoit toujours contraire le flot de la mer ne pouvant monter sy bault, c'et pourquoy nous ne pismes $y$ ariver que a force de rames, en ce temps la le montreal estoit peu babite on y voyoit qune cabpelle ou deux peres fenuites disoient la messe tous les jours et les maisons quoy que en petit nombre ne laissaient pas destre belle spatieuses et agreables celle de monsietur Le moine natif de dieppe surpassoit toutes les austres, mon camarade ducbesne diberville estant sont parent nousfusmes loger ches luy ou il nous receut en bons amis et comme gens du mesne pays. (7)}

Il regagna Dieppe, sa ville natale, en décembre de la même année.

\section{L'éclosion d'une industrie}

Pendant plusieurs décennies, seuls quelques rares touristes se sont aventurés sur nos terres. Il fallut attendre les premières années du XIX ${ }^{e}$ siècle pour réellement àssister à l'éclosion de l'industrie touristique à Montréal, comme partout ailleurs dans le monde. L'avènement de cette nouvelle forme de loisir, réservée aux mieux nantis, fut intimement liée au développement et au perfectionnement des moyens de transport. Au fur et à mesure que les déplacements s'améliorèrent, en termes de rapidité, de confort et de luxe, un nombre grandissant de riches Américains, Canadiens, Anglais et Français s'affichèrent comme touristes dans les rues de Montréal.

L'avènement des bateaux à vapeur sur le St-Laurent, dans la première décade du $\mathrm{XIX}^{\mathrm{e}}$ siècle, initièrent les premières croi- sières. Le pionnier de la navigation à vapeur sur le fleuve fut l'Accomodation, navire qui, en novembre 1809 , fit son premier trajet entre Montréal et Québec en 66 heures!

Au milieu du XIX ${ }^{c}$ siècle, le transport subit une véritable révolution. Les voyages transatlantiques entre l'Europe et l'Amérique devinrent plus accessibles et beaucoup moins risqués, et au fur et à mesure que les années avancèrent, le temps de traversée s'écourta. Dès 1850 , les réseaux de chemins de fer sillonnèrent tout l'est de l'Amérique et rapidement il ne fut «presqu'aucun endroit où le voyageur ne puisse se rendre tout en restant confortablement installé dans son fauteuil ${ }^{(5)}$. Longtemps reconnue comme deuxième ville portuaire d'Amérique du Nord, Montréal fut la plaque tournante du transport ferroviaire et la métropole canadienne du commerce et de la finance.

Tous ces progrès technologiques eurent une incidence directe sur l'expansion de la ville, surtout après la construction du pont Victoria qui confirma Montréal dans son rôle de capitale commercialedu pays. Aussi, ne faut-il surtout pas négliger le rôle prédominant qu'ont joué l'avènement de l'automobile et le développement de l'aviation civile sur l'accroissement du tourisme et des congrès à Montréal.

\section{Une hospitalité inégalée}

L'hôtellerie au Canada remonte au milieu du XVI" siècle. Les premiers établissements ouvrirent leurs portes simultanément à Montréal, Québec et Trois-Rivières. A Montréal, c'est autour de la place Royale, autrefoisle centre del'activitécommerciale de la ville, que les premières auberges s'établirent.

L'industrie hôtelière évolua peu jusqu'à l'aube du XIXe siècle. Les progrès alors réalisés au niveau des moyens de transports profitèrent grandement à l'hôtellerie montéalaise. Son développement y fut intimement rattaché puisque ces innovations stimulèrent les activités commercia- 


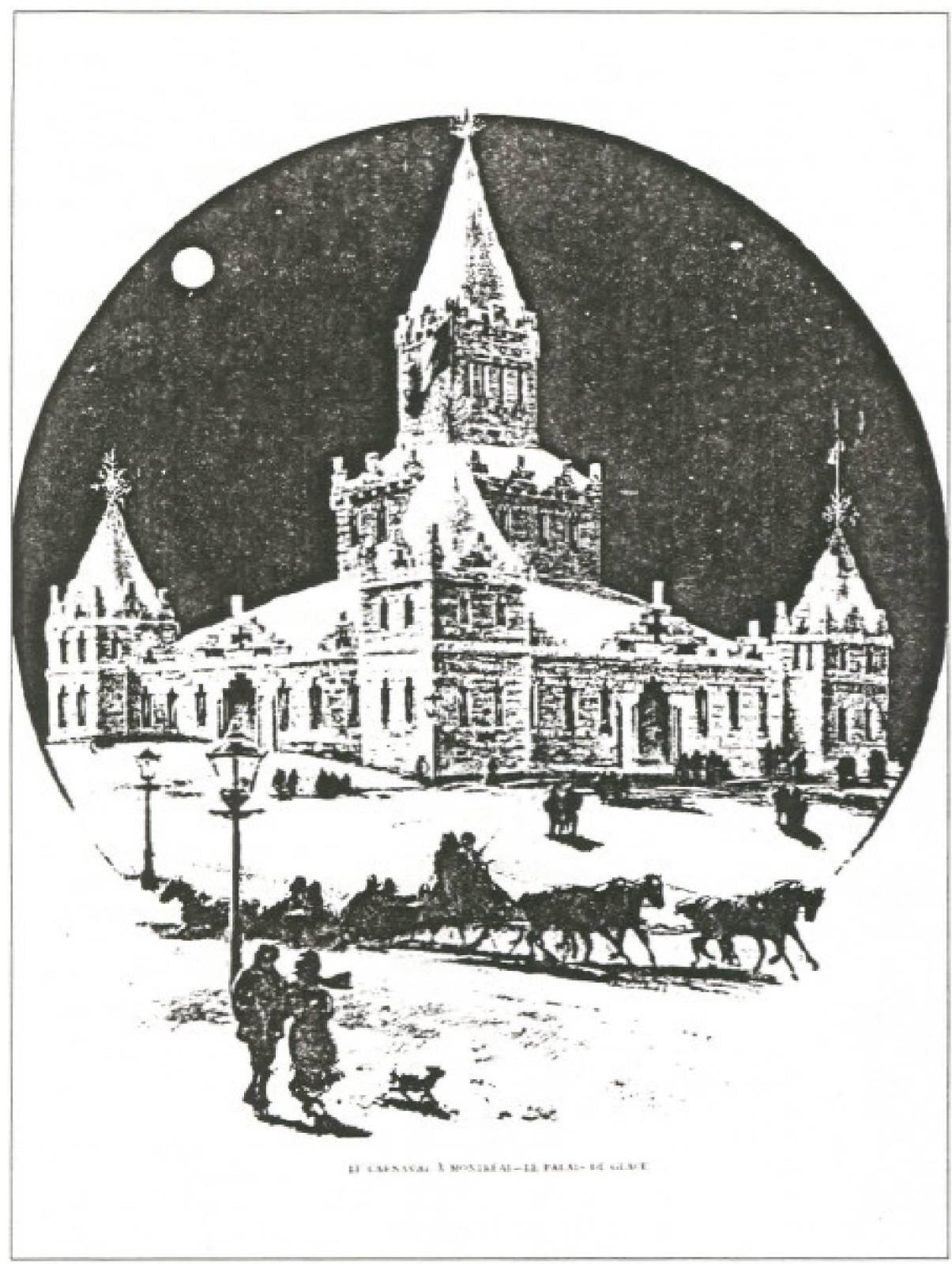

Le palais de glace de 1883 érigé sur le square Dominion. $L$ Opinjon pulique, janvier 1883. Archives du Groupe de récherchés en arts populaires, GRAP, Université du Québec à Montréal.

les de la ville et augmentèrent son achalandage.

Les premiers grands hôtels de Montréal furentl'hôtel Rasco, l'hôtel Donegana ct le St. Lawrence Hall. Leur confort et leur luxe incomparable marquèrent l'histoire hôtelière de la ville dans la première moitié du XIXe siècle.

De 1880 à 1940 , des êtablissements hôteliers exceptionnels virent le jour. S'inspirant des grands palaces de tradition européenne, la qualitê du service de ces nouveaux hôtels fut dictée par l'application de chitecrure s'inspira du célèbre Waldorf Astoria, l'un des hồtels les plus somptueux de New York.

Situé en plein coeur de l'activité commerciale de la ville, l'hottel Windsor, avec ses 400 chambres, fut pendant plusieurs années le plus vaste hôtel de l'Amérique du Nord. Il ếtait aussi le plus moderne: ascenseurs rapides, chauffage à la vapeur, eau chaude et eau froide, clochettes dans chaque chambre, etc. Les tarifs variaient entre $4 \$$ et $6 \$$ par jour.

L'histoire de l'hôtel fut malheureusement marquée par deux incendies majeurs, l'un en 1906 et l'autre en 1957 qui le détruisit presque entièrement. Néanmoins, les activités de l'hötel se poursuivirent jusqu'en 1981 dans l'annexe, la seule partie qui avait pu être sauvegardée. Aujourd'hui, le bâtiment a été recyclé en luxueux édifice à bureaux.

D'autres hôtels marquèrent cette époque glorieuse de l'hôtellerie montréalaise: I'hôtel Queen's, la gare-hôtel Viger, le Ritz-Carlton et le Mont-Royal.

L'Hôtel Ritz-Carlton est le seul établissement qui a conservé sa vocation hồtelière. Au moment de sa construction à Montréal en 1912, César Ritz, homme d'affaires français d'origine suisse et fondateur de la chaine hôteliêre, imposa deux principales conditions.

\section{En premier lieu, il fallait que le futur bôtel respecte les standards très álevés de l'bôtel Ritz : une salle de bain par chambre; des facilitếs de cuisine à chaque étage; un ball assez petit pour être intime; un grand escalier pour flatter la coquetterie de ces dames; un service de valet $24 \mathrm{~b}$ par jour et un concierge pour satisfaire les caprices de la clientèle. [...] En deturieme lieu, il fallait que les Canadiens assument la propriété de l'bôtel (*)}

Il est déplorable de constater qu'il ne reste que très peu de témoins de certe fabuleuse époque où Montréal abritait un grand nombre de fastueux hôtels. Comme en témoigne Guy Pinard :

Un des plus grands tếmoins de l'ère de cette hôtellerie de luxe fut, jusqu'a la moitié du $20^{\mathrm{E}}$ siècle, l'hôtel Windsor. Construit en 1878 en face du square Dominion (aujourd'hui square Dorchester), son ar-
De tous les bôtels construits avant 1930, à Montréal et dans la région, il n'en existe plus que 16. [...] La pauvreté de l'béritage bôtelier 
s'explique par la très grande volatilité de cette industrie forcét de plaire sans cesse aux goûts du jour et d'assister, impuissante, aux déplacements cycliques des póles d'attraction et de l'activité économigue de Montréal. De très grands bôtels de leurs époques respectives comime les bôtels St. Lawrence Hall, Ottawa, Windsor, Queen's, Viger, MontRoyal et Rasco bien sûr, sont tombés en déruétude les uns parce qu'ils n'étaient plus de leur temps, les autres parce qu'ils se trowvaient excentrés par rapport à l'épicentre économigue et touristique de la ville. Les 13 immeubles d'anciens bôtels à atuoir échappé aux pies des démolisseurs, en phus de cetux du Hency's Inn, du Rascoet du Ritz-Carltonce dernier abritant encore un bôtel - sont enur du Jacques-Cartier (ex-Nelson); du Riendeau et du StNicolas, situess Place facquesCartier; de l'Ottarsa, [...] rue StJacques; du Richelieu, [...] nue StVincent; du Bonsecours, [... ] rue StPaul est; du Queen's, [...] rue Peel; du Berkeley, du Ford, du MontRoyal et du Windsor, mieux connus des Montréalais $[. . .\}^{(5)}$

\section{Les grandes attractions}

Le statut de capitale commerciale du pays interpella plusieurs voyageurs au XLX: siècle. Tous vantèrent dans leurs récits de voyages ${ }^{(0)}$, les beautés de Montréal, le charme de ses quartiers et son ambiance. Comme l'écrivait si bien en 1903 Félix Klein:

\section{Gracieusement étendue entre la colline boisée d'où elle tire son nom et les flots bleus du Saint-Laurent; interessante comme une ville d'Europe, qu'elle semble être avec son bistoire de presque trois siècles; riche, aéré, vivante, confortable comme une ville, gu'elle est bien, de notre agge et du nouveau monde, Montreal constitue sans nal doute un des plus agréables séjours d'Amérique?}

Les lieux qu'ils fréquentaient à l'époque correspondent aujourd'hui à ce que l'on nomme le Vieux-Montréal. Un grand nombre de monuments et de bâtiments suscitèrent plusieurs commentaires de la part desvisiteurs. Hier, comme aujourd"hui, on s'imprégnait de l'animation du port ou du marché sur la place Jacques-Cartier, on appréciaitl'architecture du palais de justice, de la bourse, de l'hottel de ville et des banques, on se promenait sur le Champde-Mars, la place d'Armes, etc. La visite de l'église Notre-Dame, «l'édifice le plusvaste et le plus somptueux de toute l'Amérique du Nord ${ }^{(*)}$ constituait également une activité tuès appréciée des touristes. Jusqu'au tout début du XX's siècle, ces derniers eurent accès à l'une des tours de l'édifice religieux afin d'avoir une vue imprenable sur la ville.

Déâa à cette époque, Montrél offrait donc à ses visiteurs une multitude d'expériences et d'émotions. Que ce soit en participant à des activités récréatives, culturelles ou à des événements majeurs, Montéal pouvait toujours garantir à ses visiteurs des souvenirs inoubliables.

La descente des rapides de Lachine en bateau à vapeur, sl'une des grandes attractions d'un voyage sur le St-Laurentwos en fait preuve. C'était, pour les touristes de la fin du XIX' siècle, une aventure exaltante. Avant mëme le début de l'expédition, un sentiment d'excitation et d'appréhension sinstallait parmi les passagers. Mais leur frénésie atteignait son paroxysme lorsquu'un pilote windienw emharquait sur le bateau.

Une mise en scène impressionnante entourait son arrivée. Lorsque le navire approchait Caughnawaga, un signal était lancé. Immédiatement, un Amérindien vêtu de ses plus belles parures quittait la côte en canot vers le bateau à vapeur. II grimpait ensuite pour se rendre à la cabine de navi= gation. Cette cérémonie rassurait les passagers. Les Amérindiens avaient la réputation d'être les seuls à pouvoir réussir l'exploir de franchir les rapides de Lachine sans incidents.Aprèsla traversée des rapides, l'Amérindien allait parmi les touristes pour vendre sa photographie au coüt de 1 \$! Si l'on se fie au récit de Jules Leclercq, il semblerait que derrière ces vêtements lếgendaires se dissimulaient des hommes d'unerace autrequecellequ'ilsprétendaient être:

Cet honme se fait passer pour Indien et porte un costume a l"avenant: c'ot un moyen pas nal imaginé pour faure vendre sa photograpbie aux passagers, ce qui hui procure un joli revenu. Mais il m'avoua confidentiellentent gu'il était aussi Indien que nioi. (10)
Le peuple autochtone exer cait sur les voyageurs européens une réelle fascination. Mais un contact super ficiel à bord des navires sur les rapides de Lachine ne pouvait les satisfaire. Leur curiositéles poussait à vouloir les connaitre davantage et à les côtoyer dans leur environnement familier.

C'est ainsi qu'à la même époque, la majorité des voyageurs français visitèrent le village de Caughnawaga, aujourd'hui rebaptisé Kahnawake. Influencés par la littérature romanesque, ils croyaient en l'existence de ces personnages légendaires. Enthousiastes, ils se rendaient au village dans l'espoir d'y rencontrer les héros de leurs romans. Mais la réalité disssipait rapidement leurs illusions: à leur grande surprise, les Amérindiens vivaient de la même façon que les Blanes et les voyageurs en étaient amèrement déçus. Conscientes de l'attrait qu'exerçaient les Amérindiens sur les touristes étrangers, des entreprises organisèrent, au début du $\mathrm{XX}^{\mathrm{t}}$ siècle, des tours guidés dans le village de Caughnawaga.

Au fil des ans, plusieurs pares montréalais offrirent aux voyageurs détente et divertissement. Au XIXé siècle, des milliers de Montréalais et de touristes se rendirent au jardin Guilbault ou au parc Sohmer. Chacun proposait aux visiteurs une variété d'activités amusantes et surprenantes: acrobates, feux d'artifice, concerts, ascension en ballon, objets rares et curieux, etc.

Au tournant du siècle, quatre parcs majeurs firent la joie des touristes: leparc Dominion, l'ìle Sainte-Hélène, le parc Lafontaine et, bien sür, le parc du Mont-Royal. L'hiver fut pendant longtemps une saison touristique très achalandée à Montréal et par milliers, les touristes américains affluaient dans la ville afin de pratiquer leurs sports préférés sur la montagne: toboggan, raquette, patin, ski, etc. Comme le soulignait George A. Mc Namee, secrétaire-tuésorier du Montreal Tourist and Convention $\mathrm{Bu}$ reau (aujourd'hui OCTGM):
[...] Lessports d'biver nows ameinent un fort contingent de riches étrangers. Ils nous arrivent de New York surtout, mais aussi de Pbiladelpbie, de Wasbington, pour voir nos célebtres joutes de bockey, nos virtuoses du saut en ski; pour faire, en toboggan, ces glissades vertigi- neuses dont chez eux on parle beaucoisp... (ai) 
Durant la période estivale, de 1884 à 1918 , il fut possible d'accéder au sommet du Mont-Royal grâce à un funiculaire qui arrivait à proximité du belvédère. Sa popularité fut si grande qu'on ajouta un éclairage afin de permettre aux usagers d'en bénéficier tard le soir. Durant l'été 1885 , jusqu'a 30000 personnes profitèrent de ce service.

Montréal fut également l'hôte de nombrewx événements majeurs qui attirèrent une foule considérable de touristes. Pensons par exemple aux carnavals d'hiver, aux expositions provinciales, aux célébrations de la Saint-Jean-Baptiste, au Congrès eucharistique, à l'Exposition universelle de 1967, aux Jeux Olympiques et aux Floralies. Plusieurs de ces manifestations firent connaître Montréal à l'échelle internationale, confirmèrent son dynamisme et eurent un impact considérable sur son développement économique et urbain.

Lecarnaval d'hiver fut l'un des événements les plus marquants de la fin du XIX' siècle à Montréal. Conçu afin de promouvoir les intérêts économiques et touristaques de la ville, le premier carnaval fut créé en 1883. Les organisateurs souhaitaient réactiver l'économie dela villeen attirant des touristes durant la saison hivernale et mieux faire connaître son dynamisme auprès des voisins américains et canadiens. Montréal devait apparaître à leurs yeux comme l'une des villes les plus attrayantes du monde.

Le carnaval durait environ une semaine, à la fin du mois de janvier ou au début de février. À cette occasion, entre 50000 et 60 000 touristes américains affluaient dans la ville. Des activités sportives étaient organisées: patinage, promenades en traineau, courses de chevaux, curling..., mais l'activité préférée des Américains était incontestablement la glissade.

Les activités du carnaval d'hiver se déroulaient principalement dans l'ouest de la ville, au square Dominion et sur le MontRoyal. Toutefois, de l'animation fut aussi organisée certaines années à la place d'Armes, au Champ-de-Mars et à la place Jacques-Cartier. Plusieurs monuments de glace étaient érigés dans ces lieux publics.

Construit au square Dominion, le palais de glace était le monument le plus impressionnant du carnaval d'hiver. Le premier palais fut inauguré en janvier 1883 en présence de 3000 personnes. Édifié à l'aide de
15000 blocs de glace et de cèdre, il déploya une originalité qui ne fut jamais renouveléc.

L'attaque du palais constituait le point culminant des festivités du carnaval d'hiver. Après avoir descendu le Mont-Royal en file indienne, des centaines de raquetteurs, munisde flambeaux, se plaçaient autour du palais. Au signal de l'attaque, ils se précipitaient à l'intérieur du monument, tandis qu'une garnison tentait de défendre la forteresse. Le combat faisait rage sous les feux d'artifice pendant près d'une heure. Jusqu"à 50000 spectateurs pouvaient assister à cette cérémonic. Quant tout était terminé, les raquetteurs paradaient dans la ville et se rendaient ensuite au sommet de la montagne, où des feux d'artifice étaient encore lancés.

À ses débuts, le carnaval d'hiver de Montréal était un évếnement unique en Amérique du Nord. Mais à partir de 1885 , les Américainsorganisèrentdes manifestations semblables, ce qui entraina une baisse importante des visiteurs à Montréal. Le dernier carnaval eut lieu en 1890. Les graves problèmes financiers éprouvés depuis quelques années ne permettaient plus aux organisateurs de poursuivre leurs activités.

Ainsi, au fil des siècles, Montréal s'est toujours dévoilé̉e à l'étranger comme une ville aux multiples plaisirs. Ville de romantisme et d'histoire, destination sanségale pour les joies de l'hiver, ville d'agrément..., on la surnomma même le Paris de l'Amérique du Nord. Comme le mentionnait en 1932 GeorgeA. McNamee, secrétaire-trésorier du montreal Tourist and Convention Bureau: *[... les touristes américains viennent à Montréal parce que, auprès des leurs, notre ville est gaie; parce qu'ils y sentent une atmosphère française, quelque chose d'indéfinissable qui, de loin leur rappelle Paris $[\ldots] m^{(112)}$

\section{BIBUOGRAPHE}

COLLAAD, Edgar Andrew, Passage to the Sea, the Story of Canada Steamship Lines, Doubleday Canada Lid, Toronto, 1991

DUFAESNE, Sylvie, Le carnaval d'hiver de Montral [1883-1889]). Presses de FUniversité du Québec. Montréal, 1980

GARCEAU, Henri-Paul, Chronique de Thospitalite hôtelite du Qubbec de 1880 a 1940 : les plonniwr., Les Publications du Quebece. Ed. du Méridien, Montréal, 1990.

GREGOIRE, Jeanne, Un pare, un funiculaire, une crobx. Sociélé histórique de Montréal.
LACAOIX, Lucien, Yankese et Canadiena, impressions de voyage en Ambrique, Maison Alfred Mame et Fils, Tours, 1895

LECLEACO, Jules, Un ití en Amerique, de r'Atlantique aux montagnes Rocheuses, Ed. Plon. Nournt et Cie, imprimeurs-editeurs, Paris, 1886.

MOLSON, Du canot wu chemin de fer: histoire lllustr be dee traneports dana la province de Oubbec, Molson, Montréal, s.d.

PINARD, Guy, Montrial, son histoire, won archit tocture, Tome 2, Ed La Presse, Ottawa, 1988.

PREVOST, Robert, Le towisme au Québec, rétrospective, Oubbec-Histolr e, vol 1, no 2, avril-juin 1971. pp. 3.5 .

SIMARD, Sylvain, Mrthe et reflet de la France: image du Canada on Frane. 1850-1914. Cahiers du CACCF, Presses de I'Universte d'Ottawa, 1987.

YON, Armand, Notre premier touriste en NownelleFrance: Asseline de Ronval [1662], Lo Cahier: det Dlx, no 39, 1974, pp. 146-170.

\section{NOTES}

(1) Un manuscrit du journal de woyage d'Asseline de Ronval est canservé aux. Archives nationales du Canada.

(2) Journal en abregd des voyages de monsieur Aaseline de Ronval tant par terre que par mer, avec plusieurs remarques, circonstances of aventures tres curieunes, $1695, \mathrm{p} .50$.

(3) Sylvain SIMARD, Mythe et reflet de la France: limage du Canada en France, 1850-1914. Ca. hiers du CACCF. Presses de l'Universite d'Ottawa, 1987, p. 96.

(4) Guy Pinard, Montril, won histoire, son archl tecture. Tome 2, Ed. La Presse, Otawa, 1988, p. 246.

(5) bid., p. 158

(6) Dans le cadre de la recherche exploratoire destinée à la réalisation de l'exposition sur l'histoire du tourisme a Montréal, les récits de vayageuis fur rent une procieuse source d"information. Cette forme de littérature fut trés populaire durant la deuxieme moitie du XIX* sibcle, époque où lin. dustrie tounistique était en plein essor. Les individus qui woyageaient alors pour le plaisir devaient entierement assumer leurs frais de vovages. Its comptaient donc sur la publication de leurs récits de voyages pour rembourser une partie de leurs dépenses.

7) Sylvain SIMARD, op. cit. p. 134

(9) Lucien LACROIX, Yankees ot Canadiens, imprestions do voyages en Amérique, Maison Alfred Mame et Fils, Tours, 1895, p. 114,

(9) Jules Leclerca. Un bté en Amúrique, de I'Atlantique wx montagnes Rocheuses, Ed. Plon, Nourrit et Cie, Imprimeursediteurs, Paris, 1886, p. 357.

[10) bid., p. 357

(11) Pierre Lefort, Ce que nous raporte en movenne lindustrie du tourisme en un an, La Presse, 16 décembre 1932, p. 17 .

(12) Bid.. D. 17 